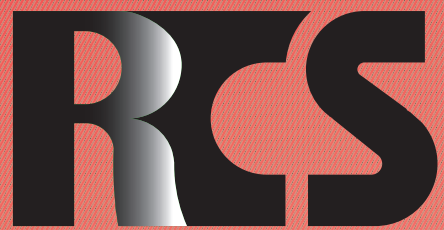

Depósito legal ppi $201502 Z U 4662$

Esta publicación científica en formato digital es continuidad de la revista impresa Depósito Legal: pp $197402 Z U 789$

- ISSN: 1315-9518 • ISSN-E: 2477-9431

Revista de Ciencias Sociales

Universidad del Zulia. Revista de la Facultad de Ciencias Económicas y Sociales Vol. XXVII. No. 2

Abril-Junio 2021

Esta publicación científica en formato digital es continuidad de la revista impresa Depósito Legal: pp $197402 Z$ Z789 ISSN: 1315-9518 


\title{
Precariedad laboral en población afrodescendiente e indígena agravada por el conflicto armado en Colombia
}

\author{
Palacios, Yennesit \\ Mondragón, Sergio ${ }^{* *}$
}

\section{Resumen}

Este artículo es fruto de un proceso investigativo que se desarrolla en el contexto del conflicto armado, diversas vulneraciones que ha padecido la comunidad afrodescendiente y los pueblos indígenas que, al ser desplazados por este fenómeno bélico, han padecido desde precariedad laboral hasta racismo estructural. De esta manera, el objetivo central de la pesquisa se centró en las afectaciones de estos grupos étnicos integrando los conceptos de raza y etnicidad, discriminación y desigualdad laboral con perspectiva étnica y de género, para visibilizar múltiples violaciones a los derechos humanos en el contexto del conflicto armado. El abordaje de las categorías se apoya en la investigación cualitativa y los resultados revelan principalmente, pese al proceso de justicia transicional, la existencia de precariedad laboral en comunidades afrodescendientes y pueblos indígenas, acentuada por la desigualdad y discriminación étnico-racial. Coyunturalmente, el marco teórico soporta que las comunidades negras y los pueblos indígenas en Colombia han padecido históricamente de dinámicas de exclusión originadas por la época de la conquista. Sin embargo, puede evidenciarse una posición de subordinación que se agrava por el conflicto armado, visibilizando un impacto desproporcionado en estas comunidades. Se concluye, que el reconocimiento del ser humano en la sociedad no puede ser estigmatizado.

Palabras clave: Etnicidad; género; conflicto armado; precarización laboral; raza.

Este artículo es un aporte reflexivo derivado de las categorías de conflicto armado, desplazamiento forzado, raza y etnicidad, en el marco del proyecto de investigación titulado: "Tensiones entre la justicia transicional y sistema de responsabilidad penal internacional: el caso de Colombia y España", asociado a la Línea de Investigación en Derechos Humanos, Género y Multiculturalismo, del Grupo Jurídico social. Texto que ha sido avalado en una versión ampliada, como capítulo de libro por la Corporación Universitaria Republicana en el texto denominado "Nuevos desafios de la investigación en derecho para poblaciones afrodescendientes indígenas y lgtbq+".

** PhD. en Derechos Humanos y Desarrollo. Docente Investigadora en la Facultad de Derecho y Ciencias Forenses, Coordinadora de la Línea de Investigación en Derechos Humanos, Género, y Multiculturalismo, del Tecnológico de Antioquia Institución Universitaria. Medellín, Colombia. E-mail: yennesit.palacios@ttdea.edu.co iD ORCID: https:// orcid.org/0000-0003-3581-439X

*** Doctorando en Seguridad Humana y Derecho Global de la Universidad Autónoma de Barcelona. Docente de Posgrados de la Institución Universitaria de Envigado (Antioquia, Colombia), adscrito al Grupo de investigación: Auditorio Constitucional. E-mail: sergio.mondragondu@unaula.edu.co iD ORCID: https://orcid.org/0000-00025189-6770

Recibido: 2020-12-06 Aceptado: 2021-02-27 


\title{
Job insecurity in Afro-descendant and indigenous populations aggravated by the armed conflict in Colombia
}

\begin{abstract}
This article is the result of an investigative process that takes place in the context of the armed conflict, various violations suffered by the Afro-descendant community and indigenous peoples who, when displaced by this war phenomenon, have suffered from job insecurity to structural racism. In this way, the central objective of the research focused on the effects of these ethnic groups, integrating the concepts of race and ethnicity, discrimination and labor inequality with an ethnic and gender perspective, to make visible multiple human rights violations in the context of the armed conflict. The approach to the categories is supported by qualitative research and the results mainly reveal, despite the transitional justice process, the existence of job insecurity in Afro-descendant communities and indigenous peoples, accentuated by ethnic-racial inequality and discrimination. Circumstantially, the theoretical framework supports that black communities and indigenous peoples in Colombia have historically suffered from dynamics of exclusion originated by the time of the conquest. However, a subordinate position can be evidenced that is aggravated by the armed conflict, making visible a disproportionate impact on these communities. It is concluded that the recognition of the human being in society cannot be stigmatized.
\end{abstract}

Keywords: Ethnicity; gender; armed conflict; job insecurity; race.

\section{Introducción}

Este estudio es fruto de reflexiones investigativas adelantadas en el marco de la Línea de Investigación en Derechos Humanos, Género y Multiculturalismo, concentradas en las comunidades afrodescendientes y pueblos indígena, desplazados por el conflicto armado. El mismo, se desarrolla a través de tres partes sustanciales que soportan la hipótesis, en relación a que, la población negra e indígena, desplazada por la violencia, sufre o padece diversas afectaciones, entre ellas, precariedad laboral y racismo, además del impacto desproporcionado y de género en el contexto del conflicto armado.

El artículo, se fundamenta en la necesidad de dar a conocer diferentes dinámicas de exclusión de comunidades negras y pueblos indígenas, las cuales reflejan desde la precariedad laboral, hasta el racismo estructural, problemática que se agrava por el conflicto armado, pese al actual proceso de justicia transicional que adelanta el Estado colombiano, por lo que la pregunta que configura la actual pesquisa corresponde a: ¿Cuáles son las condiciones de desigualdad y discriminación étnico-racial que determinan la exclusión laboral respecto de comunidades negras y pueblos indígenas, agravada por el conflicto armado interno en Colombia?

Así pues, la estructura de este opúsculo se desarrolla en función de las categorías "negro" e "indígena", desde las cuales se hace una primera deconstrucción para superar, contrahegemónicamente, el alcance despectivo de tales conceptos desde la época de la conquista hasta la actualidad (Lamus, 2012). De la misma forma, se abordan los conceptos de raza y etnicidad, que se solapan desde la asociación de factores biológicos que ofrece el primero de ellos en relación con las identidades culturales que ofrece el segundo.

En un segundo momento, se exponen 
las distintas formas de discriminación, enmarcadas en los contextos de desigualdad laboral con perspectiva de género y étnica, que se basan en la dominación sociocultural y económica que ha impedido el acceso de grupos poblacionales minoritarios al disfruto de bienes simbólicos y de consumo (Wade, 1997). Por último, una tercera fase del escrito se concentra en explicar la consolidación de desventajas históricas acumulativas, que conducen a la precarización laboral de la población negra e indígena, agravada por el conflicto armado interno.

Metodológicamente, la investigación se soporta en un enfoque cualitativo (Hernández, Fernández y Baptista 2014) y se centra en las epistemologías del sur como forma de repensar la lucha de los oprimidos, para visibilizar la voz de los silenciados históricamente bajo el poder hegemónico. Al mismo tiempo, se centra en los estudios étnicos y poscoloniales, situándose en teóricos clave, tales como: Arturo Escobar, Aníbal Quijano, Michel Yudell, Peter Wade, entre muchos otros.

El estudio permite concluir que las comunidades afrodescendientes y los pueblos indígenas, aún después de la conquista, continúan sufriendo exclusión social y discriminación. Lo cual, para el caso de Colombia, es agravado por el conflicto armado. Por otra parte, también es cierto que, la construcción de un nuevo modelo de inclusión social basado en las medidas de acciones afirmativas, conduce al acaecimiento de impactos económicos, sociales y culturales más positivos, que reducen las dinámicas de discriminación/exclusión y dominación/ subordinación de los colectivos étnicos, en el marco del conflicto armado interno, entendido este último "como una manifestación bélica en un territorio determinado, sin expandir su aplicación ajurisdicción internacional" (Vallejo y Cubides, 2017, p.34). Concretamente, en el caso colombiano el conflicto armado ha tenido la participación de diferentes actores armados, esto es, los grupos guerrillero, los paramilitares y el mismo Estado.

\section{Deconstrucción de la categoría negro e indígena}

Las categorías negro e indígena, surgen de la búsqueda de la propia identidad de sujetos de derechos que, en el pasado, producto de la conquista europea en el continente americano, fueron excluidos y tratados como no personas, de manera que, los constructos sociales actuales han reivindicado las categorías de afrodescendiente e indígena, contrahegemónicamente, precisamente, para reivindicar derechos, productos de arduas luchas sociales. Así pues, la aparición de identidades segregadas, heterodirigidas y etiquetadas (Giménez, 1997; 2005), han permitido en la lucha por los derechos humanos desde abajo, la superación y el reconocimiento de los demás como diferentes y la autoidentificación autónoma de los sujetos como diversos cultural y étnicamente.

Lo anterior, se ha visto impulsado por el reclamo por la diferencia como fórmula indignación ante lo intolerable, pues la perspectiva colonial hegemónica ha controlado el discurso de dominación/ subordinación hasta los últimos días. En efecto, tras el lastre peyorativo emergido con el discurso de las "razas", y con ello, la superioridad e inferioridad de unos grupos en relación a los otros, los afrodescendientes e indígenas en la década actual, atraviesan un despertar contrahegemónico en la lucha por el reconocimiento desde el sujeto, superando, al menos teóricamente, los estereotipos derivados de la colonia, que se han ido aboliendo desde acontecimientos como el cimarronismo o resistencia a la esclavitud, que permitió su abolición en Colombia para el año 1851 (Programa de las Naciones Unidas para el Desarrollo [PNUD], 2011; 2012).

No obstante lo anterior, han emergido nuevas formas de esclavitud y discriminación, que provienen precisamente de la conceptualización marcada que existe entre "raza" negra, indígena y blanca, conducente a la teoría de la pureza de las razas (Guzmán, 
2011). En ese orden de ideas, las opiniones eurocentristas que distinguen claramente los parches culturales y genéticos, han generado discriminación racial estructural, que se traduce en la precarización de derechos y estigmas peyorativos debido al origen étnico.

En ese entendido, se han asociado las categorías de negro, blanco e indígena a los conceptos de pobreza, desarrollo y subdesarrollo de modelos económicos y socioculturales, dejando en evidencia el clasismo y racismo en los discursos estigmatizados de gobernantes, sectores gremiales y profesionales, poco ilustrados frente al tema. Esta situación, se conoce de forma segregada como "interculturalidad" y diferencia al negro del indígena, así como a estos dos del blanco y del mestizo, bajo un concepto de sexualidad, erotismo y reproducción incontrolada, que amenaza con cercenar los derechos, violar y exterminar a la población blanca (Castro-Gómez y Grosfoguel, 2007).

Así pues, se observa cómo por ejemplo la categoría indígena se define desde el color de la piel, el lenguaje, la costumbre, la condición tribal y las reglas de vida, como una población aborigen, nativa, no civilizada, autóctona o semibárbara (Papadópolo, 1995), que carece de educación, por lo que, en el marco de la teoría lombrosiana (Mattelart, 2009), el diálogo cultural con la etnicidad es imposible, dada la superioridad de algunos colectivos sociales con relación a otros grupos que han sido subordinados históricamente (Yudell, 2014).

De esta manera, es necesario deconstruir el origen de los conceptos de negro e indígena, sin acudir a teorías de etiquetamiento racional (Giddens, 1991), por razones de odio, ira o discrepancia psicológica por desplazamiento, lo cual se suma al uso manifiestamente errado del concepto raza (Yudell, 2014), el cual realmente es producto de unas señales visuales y genéticas de diversidad humana, creadas por medio de la abstracción tanto individual como colectiva.

Así mismo, como ocurría en la antigua Grecia y Roma, donde la principal división entre las personas se daba por el carácter de ciudadano o bárbaro (Yudell, 2014), el sentimiento de exclusión humana, ha surgido también por razones del linaje o pureza de sangre, filiación religiosa o lugar de origen, como sucedió en la edad media; o por la colonización, que legalizó la esclavitud con base en criterios de superioridad e inferioridad de las personas.

En relación con la colonización, se aprecia que a través de ella se matizó el concepto de superioridad e inferioridad al de raza, pues esta última dejó de formar parte de la caracterización animal para pasar a formar parte de la clasificación humana, con base en lo precisado por autores franceses y suecos, como Louis LeClerc y Carolus Linnaeus (Yudell, 2014), quienes distinguieron a los americanos, de los asiáticos, de los africanos $\mathrm{y}$ de los europeos, por sus rasgos físicos y emocionales.

Así por ejemplo, los americanos, eran considerados como coléricos, rojizos, tiesos, de pelo negro, con orificios nasales amplios, obstinados, alegres y libres, regulados solo por la costumbre; los asiáticos, eran vistos como melancólicos, envarados, de pelo negro, ojos oscuros, severos, altivos y ávidos, colmados de opiniones; los africanos, eran concebidos como negros, flemáticos, de pelo negro, rizado, nariz prominente, labios gruesos, mujeres sin vergüenza pero amamantadoras, astutos, insensibles, indolentes, omisivos y caprichosos; y los europeos, eran considerados como blancos, temperamentales, musculosos, ojos azules, amables, regulados jurídicamente con ideología e invención (Yudell, 2014).

De esta forma, se aprecia que la división de razas superiores e inferiores ha sido un proceso de construcción ideológica, social, económica y política, que se le atribuye a los intereses de los grupos dominantes, quienes para el caso de los negros e indígenas, asumen simbólica y materialmente su inferioridad, debido a la ausencia de condiciones materiales, carencia de educación y de violencia marcada (Castro-Gómez y Grosfoguel, 2007). Es por tal razón, que Goffman (2006), ha entendido que es el mismo seno social el que categoriza 
los grupos sociales, imponiendo el concepto de status social frente al de identidad social, que debería ser mejor empleado para referirse a sujetos o colectivos sociales.

Estas ideas preliminares fundaron el eurocentrismo en el marco del capitalismo y el neoliberalismo, por lo que las diferencias sociales se enfatizaron en la distribución mundial del trabajo y el intercambio de mercancías, así como, en los modelos económicos y la formación de Estados-nación modernos (Quijano, 2017), lo cual se aunó a las segregaciones biológicas, las particularidades genéticas y las desigualdades económicas, sociales y culturales (Yudell, 2014).

Parafraseando a Castro-Gómez y Grosfoguel (2007), los negros son más aguerridos y apasionados que los indios americanos, a pesar de la falta de educación, que solo puede ser dada como sirvientes o esclavos; y los hindúes, pueden ser educados en artes, pero no las ciencias, porque su pasividad les impide lograr amplios conocimientos. De lo cual, se desprende claramente el concepto de segregación afrodescendiente e indígena, desde factores biológicos, emocionales, afectivos, espirituales, educativos y sociales, para entender que la deconstrucción étnica siempre ha gozado de estigmas e imaginarios, perpetrados en manifestaciones o acciones subjetivas y erradas, que solo conducen a la discriminación racial, desde una descontextualización ontológica.

\subsection{Binomio "raza" y etnicidad}

El intento de categorizar a los colectivos por razas, desconoce la realidad biológica de los sujetos que las integran, pues en estricto sentido no se debe hacer alusión al concepto de raza, sino de variaciones físicas humanas (Guiddens, 1991), para entender al individuo o ser humano. Por tal motivo, desde la psicología se entiende que los seres humanos pueden conservar patrones comunes en las capacidades cognoscitivas, con independencia de su origen nativo, por lo que es erróneo segregar a los individuos en negros, amerindios, blancos, africanos o europeos (Wade, 2011).

La anterior concepción, ha traído consigo el fenómeno de la negación por exclusión, el cual parte de las construcciones ideológicas esbozadas desde la conquista, en función de asumir el reconocimiento y categorización de una determinada cultura, no por sus pautas de comportamiento, sino por sus orígenes (Guiddens, 1991), que obliga a los sujetos de determinado grupo o colectivo social a no reconocerse cultural o étnicamente y generar auto discriminación, permitiendo que los grupos de raza africana o negra, como despectivamente se les ha conocido, sean excluidos de grandes proyectos socioculturales (Almario, 2001).

En efecto, la raza y la etnicidad, como categorías de análisis parten de diferencias, radicadas en los rasgos biológicos y físicos, que se solapan bajo los estigmas de la superioridad e inferioridad, los cuales generan la falta de pertenencia, cultura, desigualdad y discriminación, aunada a la prominente dominación de un grupo social respecto de otro (Bello y Rangel, 2000). Esto se suma a la postura de autores como Quijano (2000), quien reconoce que el concepto de raza parte de una categoría mental moderna, que refiere de la conquista para diferenciar a los conquistadores de los conquistados, pero que después se deconstruyó en una ausencia o falencia de estructuras biológicas por parte de los esclavizados.

Es a partir de lo anterior, que los conceptos de raza y etnia se han confundido, en la medida que el primero, se ha asociado más a características biológicas, en tanto que el segundo, se ha relacionado más con las identidades culturales, que inciden en la consolidación de un concepto más claro de etnicidad, desde la categorización social (Floya y Yuval-Davis, 1992), como un lenguaje de la geografía cultural, que revela la intención de construir un nuevo modelo de colectividades para su entendimiento en el mundo moderno (Wade, 1997).

En ese orden de ideas, la etnicidad se puede entender desde la sociobiología como un proceso de selección genética y aptitud, 
que en su extensión se hace más situacional, porque conlleva factores de actitud, precepción y sentimientos que, de forma efímera, pueden mutar o variar dependiendo de la evolución que tienen los sujetos desde su misma cultura o en las integraciones que realice con otras. Lo cual incide en el favorecimiento de intereses tanto individuales o colectivos, como arma útil, efectiva y eficaz para promover seguidores, masas o élites de grupos (Smith, 1997). Esto, unido a concepciones de otros autores como Guiddens (1991), permite entender el concepto de etnicidad, a partir de prácticas culturales y visiones antropocéntricas, que distinguen a una comunidad de personas de otras, por la lengua, la historia, la ascendencia, la religión y la vestimenta, así como también, por concepciones arcaicas de dominación, por razones de poder, astucia e inteligencia.

\subsection{Convergencia epistemológica: Etnia, raza y género}

En palabras de Stolcke (2000), "la diferencia racial se construye a través del género (...) el racismo divide la identidad y experiencia de género (...) y el género y la raza configuran la clase" (p-27-28), de manera que los conceptos de clase, raza y género, se relacionan e interactúan entre sí, pero más que de entenderlo en contextos diferenciales de hombres y mujeres, por ejemplo, lo hacen desde la constitución de desigualdad social, que fenotípicamente como ya se dijo, hace que la idea de raza se marque entre conquistadores e indios a partir de la colonia.

La idea de raza, que parte de la conquista de América, resalta las diferencias fenotípicas entre indios y conquistadores, sin embargo, el primer uso que se le dio a dicho concepto no fue respecto de los pobladores indígenas que habitaban las tierras colonizadas, sino a los esclavos provenientes de las costas africanas, a quienes se les daría el nombre de "negros" por su piel oscura (Quijano, 2000), con lo cual, se evidencian los inicios bastante primigenios de símbolos y categorías sociales creadas para desnaturalizar la esencia humana, desde la exclusión social.

En este punto, vale la pena aclarar que raza no es lo mismo que color, pues ambos conceptos han venido siendo asociados a partir de constructos e imaginarios colectivos, que lo único que han hecho desde finales del siglo XIX (Quijano, 2000), es denigrar el ser humano, desde desigualdades sociales marcadas tangencialmente; por lo que, cuando las diferencias sexuales y raciales se conectan o asemejan con los rasgos naturales, se agravan las diversas maneras de exclusión que se traducen bajo el fenómeno de la discriminación racial, como ocurre por ejemplo con el factor "estatura" que para algunos sigue siendo una causal de distinción biológica e incluso de fuerza o dominación entre hombres y mujeres (Stolcke, 2000).

A partir de lo anterior, se puede concluir para este primer apartado, que la colonización representó la implantación de un modelo socioeconómico y sociocultural, basado en la esclavitud como fuerza de trabajo, que condujo a una subordinación excluyente de afrodescendientes e indígenas, que solo produjo beneficios para los colonizadores, quienes se aprovecharon no solo de los réditos, sino también de la división social de trabajo en sí misma para reproducir las condiciones de desigualdad étnica-racial en el mercado capitalista (Viáfara, Urrea-Giraldo y Correa, 2009), lo cual muestra una distribución errada de categorías de superioridad e inferioridad marcadas, dependiendo de la raza, la intelectualidad o la aptitud de un individuo, para actuar o desenvolverse en la sociedad; siendo el hombre blanco, considerado como el científico, objetivo y racional, mientras que el hombre de color o las mujeres en general, representarían solo lo místico, lo subjetivo o lo irracional (Castro-Gómez y Grosfoguel, 2007).

A continuación se evidenciará cómo las categorías de etnia, raza y género se conectan en el marco de la precariedad laboral, la cual además de haberse originado en los procesos históricos, como los coloniales, también se consolida desde los factores de desigualdad vistos previamente, con el agravante de que 
el conflicto armado en varios países, como el colombiano, acentúa la afectación a colectivos protegidos por las Cartas Constitucionales, incluida la de 1991, especialmente a las afrodescendientes, indígenas y tribales, a partir de los desplazamientos forzados.

\section{Distintas formas de discriminación explicadas desde la desigualdad laboral con perspectiva étnica y de género}

Como se expuso previamente, los conceptos de raza y etnicidad se han arraigado culturalmente desde las construcciones sociales gestadas por el ser humano, sin embargo, al lado de estos conceptos se suman los de género y clase, que se sustentan en diversos sistemas económicos, sociales y culturales (Wade, 1997), en los que se refleja la falta de acceso equitativo a los bienes simbólicos y de consumo humano.

Un ejemplo de la inequidad de género, se presenta en la baja participación de mujeres en cargos públicos y privados, especialmente en aquellos de dirección, confianza y manejo, que sucumben ante el patriarcado instituido desde épocas históricas, incluido el periodo colonial. No obstante, el acceso a la educación ha menguado la desigualdad de género, aun cuando sigue siendo bastante marcada la adquisición de conocimientos, aptitudes y habilidades diferentes por parte de hombres y mujeres, debido a la categorización de profesiones u oficios, que se hace en razón a la condición física y sexual de los individuos (Blau y Kahn, 1994).

De esta manera, se aprecia claramente que mientras los hombres acceden a mejores salarios, oportunidades laborales $\mathrm{y}$ posiciones sociales, las mujeres siguen siendo discriminadas por su aparente falta de fuerza de trabajo, lo cual aunado al estricto o marcado cumplimiento de labores del hogar, hacen que su rol sea considerado como inferior en comparación al que cumplen los hombres. En ese mismo sentido, se puede avizorar que las tasas de participación en cargos de las mujeres blancas, son más altas en relación con las mujeres que pertenecen a minorías étnicas, y así mismo, los salarios e ingresos son superiores en relación con las de grupos poblacionales minoritarios, lo cual deja entrever que las razas y minorías étnicas se ven afectadas con la desigualdad de género, principalmente en lo que respecta a logros educativos y ocupacionales (Elmelech y Hsien-Hen, 2004).

En el caso de Colombia, se aprecia por medio de investigaciones académicas y cifras estadísticas, que los grupos poblacionales minoritarios se encuentran desprovistos de salarios y retribuciones económicas laborales justas, en comparación a quienes en condiciones igualitarias no pertenecen a ellos. Además, a pesar de haberse expedido la Ley 1496 de 2011, por medio de la cual se garantiza la igualdad salarial y de retribución laboral entre mujeres y hombres y se establecen mecanismos para erradicar cualquier forma de discriminación, no se vislumbra en dicha normatividad la incorporación de mujeres afrodescendientes e indígenas, ni tampoco un plan estratégico claro, específico y concreto de vinculación a cargos con inclusión social y enfoque de género (Departamento Administrativo Nacional de Estadística [DANE], 2019).

En otro informe rendido por Corporación Humanas Colombia (2015), se resalta el alto porcentaje de ocupación laboral por parte de varones de $51,2 \%$ en relación con las mujeres, cuya tasa de desempleo es más alta con un $15,6 \%$ en comparación con la de los hombres que es apenas del 9\%. Igualmente, haciendo un paralelo de la población económicamente activa que se halla laborando informalmente, se observa que el 51,9\% lo comprenden las mujeres afrodescendientes, en tanto que el $48,1 \%$ lo integran los hombres afrodescendientes. Así mismo, se refleja una preocupación en la obtención de cifras al respecto en el caso de poblaciones indígenas, las cuales se encuentran desprovistas de estudios estadísticos en la materia.

Lo anterior, refleja claramente la 
situación de discriminación y menoscabo de los derechos de la población indígena, que se suma a la falta de avances significativos para superar las diferencias o barreras de inserción socioeconómica entre la población afrodescendiente e indígena con la no étnica (PNUD, 2011) durante los últimos años. Este panorama se presenta especialmente en territorios del Chocó, Cauca y Guajira, que se encuentran habitados en su mayoría por minorías indígenas y afrodescendientes, las cuales cuentan con tasas de pobreza superiores al 50\% (PNUD, 2016), que se agravan con el conflicto armado interno, dado el impacto que ha tenido el ejercicio tanto de la violencia física y mental como del desplazamiento forzado en dichas zonas.

\subsection{Desventajas acumulativas históricas y precarización laboral de la población negra desplazada por el conflicto armado}

Dentro del rastreo estadístico efectuado al censo de la población afrocolombiana, afrodescendiente, negra, raizal y palenquera (NARP), se observa una discordancia de cifras, en términos de evolución de este tipo de pobladores, como quiera que para el año 2005 el DANE estimó en el censo que esta población NARP comprendía el 10, 5\% de la población total del país, pero en el Censo Nacional de Población y Vivienda (CNPV) llevado a cabo en el año 2018, este porcentaje se redujo en un $30 \%$, pasando de 4.311 .757 a 2.982 .223 habitantes NARP, lo cual puede catalogarse a la luz de diferentes movimientos sociales, como un racismo estadístico (Consejo Nacional de Paz Afrocolombiano [CONPA], 2019).

Lo anterior, se puede sumar al análisis que al respecto hicieron colectivos sociales, como el Movimiento Nacional Afrocolombiano (CIMARRON, 2004), quien en el informe del año 2004 determinó que en Colombia el 30\% de la población, equivalente a 12 millones de habitantes, es afrodescendiente, afectada por el conflicto armado interno, la pobreza, la falta de educación, la mortalidad infantil, la morbilidad, la mortalidad materna y el rechazo socio-racial (Conferencia Nacional de Organizaciones Afrocolombianas [CNOA], 2014).

Por lo que es claro, incluso por Planes Nacionales de Desarrollo de la misma población afrocolombiana, que su exclusión social está ligada a la falta de acceso a la salud, de educación, y a la falta de oportunidades para la inserción en el mercado laboral, que la tiene en analfabetismo, con altas necesidades básicas insatisfechas y en estado avanzado de discriminación (CIMARRON, 2004).

Estas características de la población afrocolombiana han repercutido en su proceso de estratificación social, desde el cual han sido catalogados como colectivos poco productivos, sin capital o recurso humano efectivo (Blau y Duncan, 1967). Sin embargo, es claro que las condiciones precarias de vida a las cuales se encuentran sometidos estos grupos poblacionales ameritan o demandan la necesidad de una implementación oportuna de políticas públicas en su favor, con eficacia (Viáfara, et al., 2009).

Ahora bien, lo expuesto antes no solo refleja la falta de oportunidades laborales y de precariedad de condiciones de vida de las comunidades afrodescendientes e indígenas en Colombia, sino también la ausencia de información estadística actualizada y verídica por parte del Departamento Administrativo Nacional de Estadística (DANE), que coloque en evidencia el porcentaje real de participación de integrantes pertenecientes a estas poblaciones, tanto hombres como mujeres, que ocupan cargos directivos en entidades públicas y privadas a nivel de todo el territorio nacional, para lograr implementar una política efectiva que conduzca a la inserción de todos los habitantes del país en el mercado laboral con un enfoque étnico y de género.

Aunado a lo anterior, se presenta el conflicto armado, que amenaza la vida de los integrantes de comunidades étnicas, tanto indígenas como afrodescendientes, desde el despojo de sus territorios, la violencia física y psicológica, y el desplazamiento forzado (Comisión Interamericana de Derechos 
Humanos [CIDH], 2008), lo cual hace que su reconocimiento como población marginada y vulnerable sea válida, a la luz de la gran pérdida de visibilidad, identidad y territorio, que se suman a la discriminación negativa propia por el solo hecho de ser indígenas, negros o afrocolombianos (Mow, 2010).

De esta manera, queda claro dentro del presente acápite que la discriminación socioracial, es un elemento determinante dentro de la estratificación social, profesional, laboral u ocupacional, lo cual tiene sentido, en la medida que son pocos los afrocolombianos que ocupan cargos importantes en el país, o son tenidos en cuenta para generar una alta producción económica estatal (CIMARRON, 2004).

Lo antes esbozado, se aprecia desde la pobreza y pobreza extrema que surge por falta de oportunidades dignas de trabajo, el impacto del conflicto armado y la crisis humanitaria que se vive en épocas difíciles (Corte Constitucional de Colombia, 2014), como la acaecida actualmente por la pandemia del COVID-19 en todo el mundo. De manera que, el trato desproporcionado, diferencial $\mathrm{y}$ de género en estas comunidades, reflejado en altas condiciones de precariedad de vida, confinamiento, discriminación, marginación estructural y de desplazamiento forzado (Corte Constitucional de Colombia, 2009), ha obligado que la Corte Constitucional declare el estado de cosas inconstitucional en el año 2004, a través de la Sentencia T-025 (Corte Constitucional de Colombia, 2004), aunque en la actualidad el mismo sigue vigente, pues el gobierno nacional no ha logrado dar una solución oportuna a tales problemáticas.

Así las cosas, se tiene que en Colombia ha sido la Alta Corte en materia constitucional la que ha reconocido como inconstitucionales las condiciones de desigualdad, discriminación y rechazo por razones de etnia o raza, en relación con la exclusión o restricción de acceso a los beneficios o servicios económicos, sociales y culturales (Sentencia T-1095) (Corte Constitucional de Colombia, 2005), por lo que es necesario, que tanto los indígenas como afrodescendientes gocen de una verdadera protección especial por parte del Estado, que a pesar de ser reconocida judicialmente, se ha quedado en el papel como letra muerta, debido a que el estado de cosas inconstitucional sigue incólume.

\section{Precariedad laboral con perspectiva étnica y de género}

Desde tiempos históricos, las comunidades indígenas y afrodescendientes han sido objeto de discriminación social y política, incluso desde lo que se conoce como premercado, en el marco de la desigualdad de oportunidades para adquirir bienes y servicios educativos, de salud, de vivienda y económicos en general, sobre todo por grupos étnicos y raciales. De manera que, retomando lo expuesto previamente, ha existido un trato diferencial en el mercado laboral para personas que son productivas, por motivos de raza, etnia y género (Viáfara, et al., 2009).

Así pues, en el contexto tanto del premercado como del mercado enfocado desde el capitalismo, ha existido una discriminación laboral que hunde a los afrocolombianos e indígenas hasta lo más profundo de la estructura de trabajo, reduciendo sus salarios e imposibilitando el acceso laboral en igualdad de condiciones con quienes no pertenecen a la comunidad étnica (Viáfara, et al., 2009). En ese sentido, las dinámicas laborales en la actualidad evidencian las condiciones claras de exclusión a las que se encuentran sometidos los pueblos afrocolombianos e indígenas en el país, con el agravante del conflicto armado interno, que empeora la falta de reconocimiento y protección de sus derechos aún más (Corporación para el Desarrollo Social Empresarial de los Pueblos Afrocolombianos [ECODESARROLLO], 2006).

De esta manera, estadísticas que han sido reflejadas por la jurisprudencia constitucional en Colombia, señalan que, con ocasión de desplazamiento forzado en el país, más del $74,2 \%$ de la población afrodescendiente e indígena perdió su estabilidad laboral, lo cual impactó en el cumplimiento de necesidades 
básicas insatisfechas, que pasaron de un $65,3 \%$ a apenas un $38,8 \%$. Además, en relación con la equidad de género, se observa en el Registro Único de Población Desplazadas (RUPD), que las mujeres afrodescendientes e indígenas obligadas a padecer o sufrir de desplazamiento forzado se encuentran laborando informalmente como independientes o formalmente como empleadas domésticas (Corte Constitucional de Colombia, 2009).

Lo anterior, permite no solo evidenciar la notoria incidencia que ha tenido el conflicto armado en la precariedad laboral de las comunidades afrodescendientes e indígenas, sino que también la falta de saberes, de capacitación, de educación y de preparación en competencias profesionales y laborales, hacen que las condiciones de vida de las poblaciones asentadas en tales territorios sean deplorables, al punto de poderse afirmar que la carencia de remuneración económica o salarial es correlativa a la falta de educación y culturización requerida para el acceso competitivo al mercado laboral por parte de sus integrantes (Asociación de Afrocolombianos Desplazados [AFRODES], 2009).

En ese orden de ideas, como bien lo presenta la jurisprudencia constitucional, únicamente el $11,4 \%$ de población afrocolombiana e indígena inscrita en el RUPD devenga un salario mínimo mensual en adelante, en tanto que el $21,8 \%$ apenas percibe un cuarto de salario mínimo, del cual los hombres representan más del $15 \%$ aproximadamente, mientras que las mujeres solo figuran con un $6 \%$ aproximadamente. Igualmente, según el RUPD, el $82,4 \%$ de la población afrocolombiana e indígena registrada no cuenta con afiliación al sistema de seguridad social por parte de sus empleadores o como independientes, siendo el indicador más alto para las mujeres con un $88,3 \%$ en comparación con el $82,4 \%$ del representado por los hombres (Corte Constitucional de Colombia, 2009).

Esta situación de discriminación económica, social y cultural solo ratifica la opresión que se ha ejercido desde tiempos remotos e históricos frente a las comunidades afrodescendientes e indígenas, especialmente, en lo que respecta a las mujeres por razones de racismo y sexismo, que dan cuenta de la diferenciación ideológica marcada de su raza y género en comparación con los hombres (Pierucci, 1999). Lo cual a su vez, coloca en evidencia la transgresión efectiva de derechos humanos a los colectivos étnicos que se han visto afectados desde sus territorios, con ocasión de la violencia armada, el desplazamiento forzado y el despojo de sus tierras (Nates, 2011).

En ese sentido, es necesario, viable y pertinente, que las instituciones públicas fortalezcan sus procesos de inclusión social, sobre todo en lo que respecta a población vulnerable, como la indígena y afrodescendiente, que además de ser discriminada por razones de su etnia y raza, su estado de indefensión se les agrava por causa del conflicto armado interno en Colombia (García, et al., 2018). Asimismo, vale la pena recordar que el fortalecimiento de las políticas de inclusión social en el marco de la inserción laboral para mitigar el desempleo, deben ser implementadas con enfoque de género (Laverde-Rojas, Gómez-Ríos y SellaménGarzón, 2020), beneficiando con equidad tanto a hombres como mujeres, sin incurrir en factores discriminatorios que cercenen o impidan el acceso al trabajo de forma oportuna $\mathrm{y}$ en condiciones igualitarias.

\section{Conclusiones}

De todo lo colegido previamente se puede concluir que el reconocimiento del ser humano en la sociedad no puede ser estigmatizado al concepto de "raza", sino de individuo real con derechos que pertenece a una sociedad multicultural, motivo por el cual, las categorías de negro, blanco e indígena corresponden a expresiones que deberían entrar en desuso o en decadencia, dada la clara mezcla de especies humanas existente a nivel mundial desde épocas históricas, incluida la colonia.

En ese sentido, es necesario apelar a la consolidación de un nuevo modelo de 
inclusión social que elimine tanto las barreras de relaciones humanas como las brechas de desigualdad económica, social y cultural, las cuales en el marco de los criterios de discriminación/exclusión y dominación/ subordinación, inciden en el no cumplimiento de necesidades básicas insatisfechas de colectivos afrodescendientes e indígenas.

Así las cosas, se evidencia que el panorama de la precariedad laboral de comunidades afrocolombianas e indígenas, aunque actualmente se adelanta un proceso de justicia transicional pionero en la historia de Colombia, aún se agrava por circunstancias derivadas del conflicto armado y esto se acentúa a su vez, por la discriminación étnico-racial que conduce a la restricción de oportunidades en el acceso al trabajo en condiciones igualitarias al resto de habitantes del país, a la explotación de la mano de obra o fuerza de trabajo, y a la percepción de un salario ínfimo e irrisorio que no colma las expectativas de trabajo, e incluso no alcanza el monto mínimo legal diario o mensual vigente, máxime la situación actual generada por el Covid-19.

Además de lo anterior, vale la pena destacar que entre los miembros de comunidades indígenas y afrodescendientes que habitan el territorio colombiano, se genera una desigualdad de género, la cual se suma a la inspirada por el resto de los pobladores, pues como se dio a conocer en las cifras estadísticas, la exclusión de grupos sociales vulnerables o minoritarios en el país, además de darse por motivos de etiqueta de raza, que desconoce la identidad de la etnia (pueblos) y de la etnicidad (cultura) de estos pueblos ancestrales, también, se acrecienta en términos de género, pues las mujeres son las que más sufren o padecen de las discriminaciones raciales, laborales, económicas, políticas y culturales en relación con los hombres.

De esta manera, se requiere implementar una cátedra de pedagogía y de derechos humanos al interior de todas las entidades, organizaciones, órganos, organismos e instituciones tanto públicas como privadas, para lograr el reconocimiento del otro a partir del autorreconocimiento del ser, el diálogo, la crítica sana, el análisis, la reflexión, el conocimiento de prácticas ancestrales y el reencuentro con elementos socioculturales antiguos. Esto contribuiría al retiro ideológico de conceptos como el de superioridad e inferioridad, aplicable de unos grupos en relación con otros, modificando el discurso hegemónico, racista y estereotipado para pasarlo a uno más actual, incluyente, justo y respetuoso de la diversidad étnica y sociocultural que existe en Colombia.

Finalmente, lo anterior conducirá a deconstruir nuevas categorías de inclusión política, económica, social, cultural y ambiental a futuro, que en palabras de Boaventura (2009) implicará "comenzar a vivir nuestra experiencia en el reverso de la experiencia de los otros" (p.363), en aras de obtener resultados distintos en materia de reconocimiento, salvaguarda y protección de los derechos humanos, sobre todo de aquellas comunidades que han permanecido mucho tiempo al margen de la igualdad.

\section{Referencias bibliográficas}

Almario, O. (2001). Tras las huellas de los Renacientes. Por el laberinto de la etnicidad e identidad de los grupos negros o "afrocolombianos" del Pacífico sur. En M. Pardo (Ed.), Acción colectiva, Estado y etnicidad en el Pacífico colombiano (pp. 15-39). Instituto Colombiano de Antropología e Historia.

Asociación de Afrocolombianos Desplazados - AFRODES (2009). Los derechos humanos en los afrocolombianos en situación de desplazamiento forzado. Informe presentado al Comité para la Eliminación de la Discriminación Racial (CERD). AFRODES. https:// tbinternet.ohchr.org/Treaties/CERD/ Shared\%20Documents/COL/INT CERD_NGO_COL 75 8432_E.pdf 
Bello, A., y Rangel M. (2000). Etnicidad, "raza" y equidad en América Latina y el Caribe. CEPAL. https://repositorio. cepal.org/handle/11362/31450

Blau, F. D., y Kahn, L. M. (1994). Rising wage inequality and the U.S. gender gap. American Economic Review, 84(2), 23-28.

Blau, P. M., y Duncan, O. D. (1967). The American occupational structure. John Wiley \& Sons

Boaventura, S. (2009). Una epistemología del sur: La reinvención del conocimiento y la emancipación social. Consejo Latinoamericano de Ciencias SocialesCLACSO y Grupo Editorial Siglo Veintiuno.

Castro-Gómez, S., y Grosfoguel, R. (Eds.) (2007). El giro decolonial: Reflexiones para una diversidad epistémica más allá del capitalismo global. Siglo del Hombre Editores.

Comisión Interamericana de Derechos Humanos - CIDH (2008). Informe Anual de la Comisión Interamericana de Derechos Humanos 2008. Capitulo IV (continuación). https://www.cidh. oas.org/annualrep/2008sp/cap4. Colombia.sp.htm

Conferencia Nacional de Organizaciones Afrocolombianas - $\quad$ CNOA (2014). Visibilidad estadística del pueblo afrocolombiano. CNOA. https://convergenciacnoa.org/ visibilidad-estadistica-del-puebloafrocolombiano/

Consejo Nacional de Paz Afrocolombiano CONPA (14 de noviembre de 2019). El crimen del DANE: El genocidio estadístico de la gente negra, afrocolombiana, raizal y palenquera en Colombia. Renacientes. https:// renacientes.net/blog/2019/11/15/ el-crimen-del-dane-el-genocidioestadistico-de-la-gente-negra- afrocolombiana-raizal-y-palenqueraen-colombia/

Corporación Humanas Colombia (2015). Situación de las mujeres afrocolombianas e indigenas: Colombia 2011 - 2014. Ediciones Ántropos.

Corporación para el Desarrollo Social y Empresarial de los Pueblos Afrocolombianos ECODESARROLLO (2006). Caracterización de la población afrocolombiana desplazada lineamientos de política de atención diferenciada. ECODESARROLLO.

Corte Constitucional de Colombia (2004). Sentencia T-025 de 22 de enero de 2004. Magistrado Presidente. Manuel José Cepeda Espinosa. https:// www.corteconstitucional.gov.co/ relatoria/2004/t-025-04.htm

Corte Constitucional de Colombia (2005). Sentencia T-1095 de 26 de octubre de 2005. Deber de Solidaridad. Magistrado Presidente. Clara Inés Vargas Hernández. https:// www.corteconstitucional.gov.co/ relatoria/2005/T-1095-05.htm

Corte Constitucional de Colombia (2009). Seguimiento a la Sentencia T-025 de 2004. Magistrado Presidente. Manuel José Cepeda Espinosa. Auto 005 del 26 de enero de 2009. https://www. corteconstitucional.gov.co/T-025-04/ A2009.php

Corte Constitucional de Colombia (2014). Sala Especial de Seguimiento a la Sentencia T-025 de 2004. Magistrado Presidente. Luis Ernesto Vargas Silva. Auto 073 del 27 de marzo de 2014. https://www.corteconstitucional. gov.co/T-025-04/AUTOS\%20 $2014 / 003 . \% 20$ Auto $\% 20073 \% 20$ de $\% 2027 \% 20 \mathrm{de} \% 20 \mathrm{marzo} \% 20$ de $\% 202014 . \% 20 \mathrm{Medidas} \% 20$ de $\% 20$ protecci $\%$ C $3 \%$ B 3 n $\% 20$ 
Precariedad laboral en población afrodescendiente e indígena agravada por el conflicto armado en Colombia

y $\% 20$ prevenci $\%$ C $3 \%$ B 3 n $\% 20$ para $\% 201$ as $\% 20$ comunidades $\% 20$ afrodescendientes $\% 20$ de $\% 20$ Nari\%C3\%B1o.pdf

Departamento Administrativo Nacional de Estadística - DANE (2019). Población Negra, Afrocolombiana, Raizal y Palenquera (NARP). Resultados del Censo Nacional de Población y Vivienda 2018. DANE. https://www.dane.gov.co/files/ investigaciones/boletines/gruposetnicos/presentacion-grupos-etnicospoblacion-NARP-2019.pdf

Elmelech, Y., y Hsien-Hen L. (2004). Race, ethnicity, and the gender-poverty gap. Social Science Research, 33(1), 1-27. https://doi.org/10.1016/S0049089X(03)00044-9

Floya A., y Yuval-Davis, N. (1992). Racialized boundaries: Race, nation, gender, colour and class and the anti-racist struggle. Routledge.

García, J., Durán, S. E., Parra, M. A., y Martínez, H. (2018). Inserción, integración y equidad en el ámbito laboral: Escenario empresarial posconflicto en Colombia. Revista de Ciencias Sociales (Ve), XXIV(3), 3649.

Giménez, G. (1997). Materiales para una teoría de las identidades sociales. Frontera Norte, 9(18), 9-28.

Giménez, G. (2005). Territorio e identidad. Breve introducción a la geografía cultural. Trayectorias, VII(17), 8-24.

Goffman, E. (2006). Estigma: La identidad deteriorada. Amorrortu Editores.

Guiddens, A. (1991). Sociología. Alianza Editorial

Guzmán, O. (2011). La diversidad étnica como variable en la intervención del Trabajo Social Trabajo Social, (13), 171-180. https://revistas.unal.edu.co/index.php/

\section{tsocial/article/view/28447/39617}

Hernández, S., Fernández, C., y Baptista, M. D. P. (2014). Metodología de la investigación. McGraw-Hill.

Laverde-Rojas, H., Gómez-Ríos, J. J., y Sellamén-Garzón, A. (2020). Género y grupo étnico: Condicionantes de la pobreza infantil en Colombia. Revista de Ciencias Sociales (Ve) XXVI(2), 69-94. https://doi.org/10.31876/rcs. v26i2.32425

Lamus, D. (2012). Raza y etnia, sexo y género: El significado de la diferencia y el poder. Reflexión Política, 14(27), 6884.

Mattelart, A. (2009). Un mundo vigilado. Ediciones Paidós Ibérica, S. A.

Movimiento Nacional Afrocolombiano CIMARRON (2004). Informe del Movimiento Nacional Afrocolombiano CIMARRON sobre la situación de derechos humanos de la población afrocolombiana (1994-2004). Universidad del Rosario. http:// www.urosario.edu.co/jurisprudencia/ catedra-viva-intercultural/ Documentos/informecimarron.pdf

Mow, J. (2010). Población Afrocolombiana/ Negra, Palenquera y Raizal y Derechos Humanos. Indepaz. http:// centromemoria.gov.co/wp-content/ uploads/2013/11/MDULO 4 pdf.pdf.

Nates, B. (2011). Soportes teóricos y etnográficos sobre conceptos de territorio. Co-Herencia, 8(14), 209 229.

Papadópolo, M. (1995). El nuevo enfoque internacional en materia de derechos de los pueblos indígenas. Universidad Rafael Landívar. Instituto de Investigaciones Económicas y Sociales (IDIES).

Pierucci, A. F. (1999). Ciladas da diferença. Tempo Social, 1(1), 7-33. 
Programa de las Naciones Unidas para el Desarrollo - PNUD (2011). Afrocolombianos, sus territorios $y$ condiciones de vida. PNUD-Cuaderno del Informe de Desarrollo Humano Colombia. . https://bit.ly/3bdST21

Programa de las Naciones Unidas para el Desarrollo - PNUD (2012). Los afrocolombianos frente a los objetivos de desarrollo del milenio. PNUD. http://www.undp.org/content/dam/ colombia/docs/ODM/undp-coodmafrocolombianos-2012.pdf.

Programa de las Naciones Unidas para el Desarrollo - PNUD (2016). Objetivos de desarrollo sostenible, Colombia. Herramientas de aproximación al contexto local. PNUD. http:// www.co.undp.org/content/dam/ colombia/docs/ODM/undp-coODSColombiaVSWS-2016.pdf.

Quijano, A. (2000). Colonialidad del poder, eurocentrismo y América Latina. En E. Lander (ed.), La colonialidad del saber: Eurocentrismo y ciencias sociales perspectivas latinoamericanas (pp. 201-246). CLACSO. Consejo Latinoamericano de Ciencias Sociales.

Quijano, A. (2017). ¡Qué tal raza! En R. Campoalegre y K. A. Bidaseca (Eds.), Más allá del decenio de los pueblos afrodescendientes (pp. 17-26). CLACSO. Consejo Latinoamericano de Ciencias Sociales.

Smith, A. D. (1997). La identidad nacional. Trama Editorial.
Stolcke, V. (2000). ¿Es el sexo para el género lo que la raza para la etnicidad...y la naturaleza para la sociedad? Política y Cultura, (14), 25-60.

Vallejo, H. M., y Cubides, J. (Eds.) (2017). El conflicto armado interno en Colombia: Análisis jurídico de protección a las víctimas y del medio ambiente. Escuela Superior de Guerra "General Rafael Reyes Prieto".

Viáfara, C. A., Urrea-Giraldo, F., y Correa, J. B. (2009). Desigualdades sociodemográficas y socioeconómicas, mercado laboral y discriminación étnico-racial en Colombia: Análisis estadístico como sustento de Acciones Afirmativas a favor de la población afrocolombiana. En C. M. RoseroLabbé y R. E. León (Eds.), Acciones afirmativas y ciudadanía diferenciada étnico-racial negra, afrocolombiana, palenquera y raizal. Entre Bicentenarios de las independencias y Constitución de 1991 (pp. 153-346). Universidad Nacional de Colombia, Facultad de Ciencias Humanas, Centro de Estudios Sociales - CES.

Wade, P. (1997). Race and ethnicity in Latin America. Pluto Press

Wade, P. (2011). Raza y naturaleza humana. Tabula Rasa, (14), 205-226.

Yudell, M. (2014). Breve historia del concepto raza. Pasajes: Revista de Pensamiento Contemporáneo, (44), 32-47. 